\title{
Importancia de alteración cromosómica en parejas, como causa de aborto recurrente. Revisión sistemática de la literatura
}

\author{
Alfredo Núñez S. ${ }^{1}$, Juan Enrique Schwarze M. $M S c^{2,3}$, Sonia Villa $V^{3}$, Ricardo \\ Pommer T. 3,4 \\ ${ }^{1}$ Programa de Formación de Especialistas, ${ }^{2}$ Departamento Clínico de Obstetricia y Ginecología, Universidad de \\ Santiago. ${ }^{3}$ Unidad Medicina Reproductiva Clínica Monteblanco. ${ }^{4}$ Instituto de Investigación Materno Infantil de la \\ Universidad de Chile. Santiago, Chile.
}

\section{RESUMEN}

Objetivo: Determinar la frecuencia de alteraciones genéticas en uno o ambos progenitores de parejas con aborto recurrente. Método: Se buscaron artículos en inglés en las bases de datos MEDLINE y PUBMED entre los años 1990 y 2013 que reportaran estudio de cariograma en parejas con aborto recurrente y que no se hubieran sometido a técnicas de reproducción asistida. Resultados: La frecuencia de alteraciones cromosómicas fue variable en los distintos trabajos analizados con variaciones importantes en el número de pacientes incluidos. Aunque los trabajos analizados usaron diferentes números para definir el aborto recurrente, no hubo diferencias en la frecuencia de alteraciones genéticas al considerar 2 ó más abortos versus 3 ó más. La translocación recíproca fue la alteración más frecuentemente encontrada. Las alteraciones cromosómicas fueron más frecuentes en mujeres que en hombres. Conclusión: El análisis cromosómico es fundamental en el estudio de las parejas con aborto recurrente. En aquellas parejas portadoras de alguna alteración, es esencial que se realice un consejo genético adecuado y se debe sugerir la realización de diagnóstico prenatal en embarazos futuros.

\section{PALABRAS CLAVE: Envejecimiento de la mujer, fertilidad, aborto recurrente, cariograma, consejo genético}

\section{SUMMARY}

Objective: To determine the frequency of genetic alterations in couples with recurrent miscarriage. Methods: We searched articles in English in MEDLINE and PUBMED databases between 1990 and 2013, which reported karyotype analysis in couples with recurrent miscarriage and that had not been subjected to assisted reproduction techniques. Results: The frequency of chromosomal alterations differ among different studies, furthermore we found a significant variation in the number of patients included. Although articles studied used different numbers to define recurrent miscarriage, there were no significant differences in the frequency of genetic alterations among couples with at least 2 or at least 3 miscarriages. The reciprocal translocation alteration was the most frequently found genetic alteration. Chromosomal alterations were more frequent in women. Conclusion: Chromosomal analysis is essential in the study of couples with recurrent miscarriage. In those couples carriers of some alteration, is essential to perform a genetic counseling and should be offered prenatal diagnosis in future pregnancies.

KEY WORDS: Female aging, fertility, recurrent miscarriage, karyotype analysis, genetic counseling 


\section{INTRODUCCIÓN}

Se define aborto recurrente como la pérdida espontánea y consecutiva de tres o más embarazos antes de la viabilidad o con peso fetal menor a 500 g (1). Se estima su prevalencia entre el 0,5 al $3 \%$ de las parejas en edad reproductiva (2). Esta condición muchas veces presenta un dilema tanto para la pareja como para su médico tratante: puede a llegar a ser muy angustiante para la pareja, y causar frustración al médico por la falta de un tratamiento definitivo.

Las principales causas de aborto recurrente son alteraciones las genéticas en algún miembro de la pareja, malformaciones anatómicas del útero, trombofilias, enfermedades maternas y otras. Sin embargo, en solamente la mitad de los casos se logra identificar la etiología (3).

Si uno de los progenitores es portador de una anomalía cromosómica estructural, el embrión puede tener un cariotipo normal, el cariotipo del progenitor portador, o un cariotipo no balanceado. Este último puede llevar a aborto recurrente, muerte fetal intrauterina o el nacimiento de un niño con malformaciones congénitas (4).

La frecuencia de alteraciones genéticas, tanto estructurales como mutaciones monogenéticas, aumentaría de $0,7 \%$ en la población general a 2,2\% en la población que experimentó un aborto, 4,8\% con dos abortos y $5,2 \%$ en la con tres abortos $(5,6)$. El objetivo de esta revisión es determinar la frecuencia de alteración genética en alguno de los progenitores en el caso de aborto habitual.

\section{MATERIAL Y MÉTODO}

Se buscaron artículos en las bases de datos MEDLINE y PUBMED. Los criterios de búsqueda fueron amplios y correspondieron a la presencia en cualquier campo de las palabras claves "recurrent miscarriage", "repeated miscarriage", "recurrent spontaneous abortions", "recurrent pregnancy loss", "chromosomal abnormalities", y "parental karyotype". Luego se buscaron nuevos artículos dentro del listado de referencias de cada artículo. La cobertura temporal fue desde 1990 hasta marzo de 2013.

Los criterios de inclusión fueron publicaciones en inglés que reportaran resultados de estudio de cariograma en ambos miembros de una pareja con aborto recurrente, definido como la presencia de tres o más pérdidas recurrentes. Los criterios de exclusión fue el uso de técnicas de reproducción asistida.

\section{RESULTADOS}

Luego de aplicar los criterios de búsqueda encontramos un total de 128 artículos, sólo ocho cumplieron con los criterios de inclusión (Tabla I).

De Braekeleer y Dao (4), en un estudio transversal, publicaron en 1990 su experiencia con informa- ción obtenida de la base de datos citogenéticos de parejas con aborto recurrente de la Universidad de Quebec. Se incluyó a 16.721 parejas con al menos dos abortos. Los autores encontraron que el $4,7 \%$ de las parejas tenían alguna alteración cromosómica. Las alteraciones genéticas encontradas fueron: 409 (1,4\%) con translocación recíproca, 183 (0,6\%) con translocación Robertsoniana, 59 (0,2\%) con inversión, $31(0,1 \%)$ con aneuploidía de cromosoma sexual y $7(0,02 \%)$ con cromosoma supernumerario. Los autores encontraron una relación significativa entre el tipo de alteración genética y el número de abortos registrados. Es así como, en el caso de translocación Robertsoniana el 1,38\% de las parejas con dos abortos y 1,51\% para individuos con 3 o más abortos, presentaron esta condición. En el caso de translocación recíproca, el $0,62 \%$ de parejas con dos abortos y el $0,66 \%$ de parejas con tres o más abortos presentó esta anomalía $(p<0,05)(4)$.

Sugiura-Ogasawara y cols (7), publicaron en 2004 un estudio retrospectivo en el que 100 de 1.284 parejas $(7,8 \%)$ con 2 o más abortos, presentaron un cariotipo anormal. Presentaron cariotipo anormal 34 hombres y 68 mujeres. Dos parejas presentaron cariotipo anormal en ambos padres. Del total de parejas analizadas, hubo 47 (1,8\%) con translocación recíproca, $11(0,4 \%)$ con translocación Robertsoniana y $32(1,2 \%)$ con inversión. Asimismo, los casos de parejas con translocación recíproca en cualquiera de los padres abortan significativamente más frecuentemente que las parejas con cariotipo normal.

Goddijn y cols (8), publicaron en 2004 un estudio de cohorte histórica analizando el cariotipo de parejas con dos o más abortos sucesivos. De un total de 2.648 individuos $26(1,0 \%)$ presentaron translocación recíproca, $9(0,3 \%)$ inversión, $3(0,1 \%)$ translocación Robertsoniana, $2(0,08 \%)$ aneuploidía de cromosoma sexual y $1(0,04 \%)$ con marcador cromosómico. No se encontró diferencia significativa en la edad materna entre parejas portadoras y no portadoras de anormalidad cromosómica. Además fue más frecuente la portación de alteración cromosómica en mujeres que en hombres.

Franssen y cols (9), publicaron su experiencia en 2005, usando la base de datos de seis centros de genética clínica. De un total de 11.971 parejas con al menos dos abortos, $279(2,3 \%)$ parejas eran portadoras de alguna alteración cromosómica. Las alteraciones cromosómicas descritas fueron: 174 $(0,7 \%)$ translocaciones recíprocas, $44(0,2 \%)$ translocaciones Robertsonianas, 42 inversiones $(0,2 \%)$, $7(0,03 \%)$ cromosoma marcado y otras $12(0,05 \%)$. Además este trabajo demostró que una baja edad materna al momento del segundo aborto, una historia de 3 o más abortos, historia de 2 o más abortos en hermanos y padres de las parejas, aumenta la probabilidad de ser portador de una alteración cromosómica estructural. 
Tabla I

RESUMEN DE TRABAJOS EVALUADOS

\begin{tabular}{|c|c|c|c|c|}
\hline Referencia & Tipo estudio & Criterios & Estudio cromosómico & Conclusión \\
\hline De Braekeleer (4) & Transversal & $\begin{array}{l}2 \text { o más } \\
\text { abortos }\end{array}$ & $\begin{array}{l}\text { Recomendación } \\
\text { ISCN (1981 }\end{array}$ & $\begin{array}{l}4,7 \% \text { de las parejas tienen al- } \\
\text { teración cromosómica. Hubo } \\
\text { relación entre la incidencia de } \\
\text { translocación Robertsoniana y } \\
\text { translocación recíproca con el } \\
\text { número de abortos }(p<0,05)\end{array}$ \\
\hline Sugiura-Ogasawara (7) & Retrospectivo & $\begin{array}{l}2 \text { o más } \\
\text { abortos }\end{array}$ & $\begin{array}{l}\text { Técnica estándar de } \\
\text { bandeo G }\end{array}$ & $\begin{array}{l}7,8 \% \text { de las parejas tienen alte- } \\
\text { ración cromosómica. Los casos } \\
\text { de parejas con translocación } \\
\text { recíproca en cualquiera de los } \\
\text { padres abortan más frecuente- } \\
\text { mente que las parejas con ca- } \\
\text { riotipo normal }(p<0,000000005)\end{array}$ \\
\hline Goddijn (8) & $\begin{array}{l}\text { Cohorte histórica } \\
\text { Caso control }\end{array}$ & $\begin{array}{l}2 \text { o más } \\
\text { abortos }\end{array}$ & $\begin{array}{l}\text { CLSP, procesados } \\
\text { con bandeo G }\end{array}$ & $\begin{array}{l}3,8 \% \text { de las parejas tienen alte- } \\
\text { ración cromosómica, siendo la } \\
\text { translocación recíproca la más } \\
\text { frecuente. No hubo diferencia } \\
\text { significativa en la edad materna } \\
\text { entre parejas portadoras y no } \\
\text { portadoras de la anormalidad } \\
\text { cromosómica }\end{array}$ \\
\hline Franssen (9) & Caso control & $\begin{array}{l}2 \text { o más } \\
\text { abortos }\end{array}$ & $\begin{array}{l}\text { Recomendación } \\
\text { ISCN (1995) }\end{array}$ & $\begin{array}{l}2,3 \% \text { de las parejas tienen alte- } \\
\text { ración cromosómica, transloca- } \\
\text { ción recíproca la más frecuente }\end{array}$ \\
\hline Elghezal (10) & Retrospectivo & $\begin{array}{l}3 \text { o más } \\
\text { abortos }\end{array}$ & $\begin{array}{l}\text { CLSP, procesados } \\
\text { con bandeo } \mathrm{R}\end{array}$ & $\begin{array}{l}6,9 \% \text { de las parejas tienen al- } \\
\text { teraciones cromosómicas. Mu- } \\
\text { jeres } 5,2 \% \text { y hombres } 1,7 \% \text {, } \\
\text { (p<0.001). Translocaciones } \\
\text { reciprocas }(1 \%) \text {, inversiones } \\
(0,6 \%) \text {, translocaciones Ro- } \\
\text { bertsonianas }(0,4 \%)\end{array}$ \\
\hline Pal (11) & Retrospectivo & $\begin{array}{l}2 \text { o más } \\
\text { abortos }\end{array}$ & $\begin{array}{l}\text { CLSP, procesados } \\
\text { con bandeo GTG. } \\
\text { ISCN } 205\end{array}$ & $\begin{array}{l}8,9 \% \text { de las parejas tienen al- } \\
\text { teraciones cromosómicas, } \\
\text { translocación recíproca la más } \\
\text { frecuente }\end{array}$ \\
\hline Niroumanesh (12) & Descriptivo & $\begin{array}{l}2 \text { o más } \\
\text { abortos }\end{array}$ & $\begin{array}{l}\text { CLSP, procesados } \\
\text { con bandeo GTG. } \\
\text { ISCN } 205\end{array}$ & $\begin{array}{l}12 \% \text { de las parejas tiene alte- } \\
\text { ración cromosómica, transloca- } \\
\text { ciones recíprocas e inversiones } \\
\text { las más frecuentes }\end{array}$ \\
\hline Zhang (13) & Retrospectivo & $\begin{array}{l}3 \text { o más } \\
\text { abortos }\end{array}$ & $\begin{array}{l}\text { CLSP, procesados } \\
\text { con bandeo GTG. }\end{array}$ & $\begin{array}{l}3 \% \text { de las parejas tienen alte- } \\
\text { ración cromosómica, translo- } \\
\text { caciones recíprocas las más } \\
\text { frecuentes }\end{array}$ \\
\hline
\end{tabular}

CLSP: Cultivo de linfocitos en sangre periférica. ISCN: International Standing Committe on Human Cytogenetic Nomenclature. 
Elghezal y cols (10), publicaron en 2007 un estudio retrospectivo en un total de 1.400 parejas con 3 o más abortos. Encontraron que 97 (6,9\%) tenían alguna alteración cromosómica. Se detectó translocaciones recíprocas en 29 pacientes (1\%), inversiones en 16 pacientes $(0,6 \%)$, con inversiones y translocaciones Robertsonianas en 12 pacientes $(0,4 \%)$. Las mujeres son más afectadas que los hombres con una prevalencia de $5,21 \%$ y $1,71 \%$, respectivamente $(p<0,001)$. Además se demostró que la prevalencia de alteraciones cromosómicas no tiene relación con el número de abortos.

Pal y cols (11), en 2009 publicaron un estudio retrospectivo sobre un total de 56 parejas derivadas por dos o más abortos; 5 parejas (8,9\%) presentaron alteraciones cromosómicas. De un total de 112 pacientes, $3(2,7 \%)$ presentaron translocación recíproca, $1(0,9 \%)$ translocación Robertsoniana y 1 (0,9\% con alteración numérica). Además, se observó que la frecuencia de un hijo sano posterior a los abortos, no tiene diferencia significativa entre parejas con cariotipo normal y alteraciones cromosómicas.

Niroumanesh y cols (12), en 2011 comunicaron un estudio descriptivo en 100 parejas con 2 o más abortos. Encontraron que 12 parejas (12\%) presentaban alteración cromosómica; 8 mujeres y 5 hombres presentaron alteración cromosómica (una pareja con alteración cromosómica en ambos). La prevalencia de alteraciones cromosómicas fue distribuida de la siguiente manera: 4 pacientes (2\%) presentaron translocación recíproca, 4 pacientes $(2 \%)$ con inversión, 3 pacientes $(1,5 \%)$ con translocación Robertsoniana, 1 paciente $(0,5 \%)$ con marcador cromosómico y 1 paciente $(0,5 \%)$ con polimorfismo.

Zhang y cols (13), publicaron en 2011 un estudio retrospectivo con pacientes que presentaron 3 o más abortos. En un total de 734 pacientes encontraron un 3\% de alteraciones cromosómicas (22 pacientes), 14 mujeres $(1,91 \%)$ y 8 hombres $(1,09 \%)$. Se encontró 18 pacientes $(2,45 \%)$ con translocación recíproca, 2 pacientes $(0,27 \%)$ con translocación Robertsoniana, 1 paciente $(0,14)$ con inversión y 1 paciente $(0,14 \%)$ con anormalidad dicéntrica.

\section{DISCUSIÓN}

En esta revisión sistemática buscamos determinar la frecuencia de alteraciones genéticas en parejas con diagnóstico de aborto recurrente. La alteración cromosómica, en alguno de los padres, causa aborto recurrente.

De los 8 trabajos que cumplieron los criterios de selección, 6 definieron aborto recurrente como la presencia de al menos dos abortos, mientras que los otros 2 ocuparon la definición más tradicional de la presencia de al menos tres abortos (1).

En los 6 trabajos que definieron aborto recurrente como la presencia de al menos dos abortos, la frecuencia de alteraciones genéticas en los padres fue entre 2,3 y $12 \%$. En cambio, los 2 trabajos que definieron la presencia de aborto recurrente como la presencia de al menos 3 aborto, la frecuencia de alteraciones cromosómicas en los padres fue entre 3,0 y $6,9 \%$.

Sin embargo, con ambas definiciones, la alteración genética más frecuente fue la translocación, y las alteraciones cromosómicas fueron más frecuentes en mujeres que en hombres $(4,10)$. Es interesante destacar, que incluso en parejas con 2 abortos presentan una alta frecuencia de anomalías genéticas, por lo que se podría sugerir solicitar precozmente el estudio genético en parejas con dos abortos.

La principal debilidad de los estudios realizados es la diferencia entre el número de pacientes analizados. Es así como en el estudio de De Braekeleer y Dao (4), analizaron un total de 29.096 pacientes, encontrando una frecuencia de $2,3 \%$ de alteraciones cromosómicas en los pacientes analizados (4,7\% de las parejas), con un intervalo de confianza del $95 \%$ del $2,2 \%$ a $2,5 \%$. En cambio el estudio de $\mathrm{Pal}$ y cols (11), analizaron solamente 112 individuos (56 parejas), encontrando una frecuencia de $8,9 \%$ de anomalías cromosómicas en los pacientes, con un intervalo de confianza del $95 \%$ de $4,3 \%$ a $15,8 \%$.

\section{CONCLUSIÓN}

Independientemente de la definición usada, un consejo genético adecuado es esencial en la evaluación de parejas con aborto recurrente, por lo cual, el análisis cromosómico debe ser una parte fundamental del estudio de éstas parejas. No hay mayor diferencia en los resultados con dos o tres abortos, por lo que sería recomendable realizar estudio genético en parejas una vez ocurrido el segundo aborto. La importancia del estudio genético es que permite realizar una adecuada consejería genética para la pareja, y eventualmente, realizar diagnóstico genético preimplantacional o prenatal.

\section{REFERENCIAS}

1. Stirrat GM. Recurrent miscarriage. Lancet 1990;336(8716):673-5.

2. Li TC, Makris M, Tomsu M, Tuckerman E, Laird S. Recurrent miscarriage: aetiology, management and prognosis. Hum Reprod Update 2002;8(5):463-81.

3. Clifford K, Rai R, Regan L. Future pregnancy outcome in unexplained recurrent first trimester miscarriage. Hum Reprod 1997;12(2):387-9.

4. De Braekeleer M, Dao TN. Cytogenetic studies in couples experiencing repeated pregnancy losses. Hum Reprod 1990;5(5):519-28.

5. Carrington B, Sacks G, Regan L. Recurrent miscarriage: pathophysiology and outcome. Curr Opin Obstet Gynecol 2005;17(6):591-7. 
6. Hook EB, Healy NP, Willey AM. How much difference does chromosome banding make? Adjustments in prevalence and mutation rates of human structural cytogenetic abnormalities. Ann Hum Genet 1989;53(Pt 3):237-42.

7. Sugiura-Ogasawara M, Ozaki $Y$, Sato T, Suzumori N, Suzumori K. Poor prognosis of recurrent aborters with either maternal or paternal reciprocal translocations. Fertil Steril 2004;81(2):367-73.

8. Goddijn M, Joosten JH, Knegt AC, van derVeen F, Franssen MT, Bonsel GJ, Leschot NJ. Clinical relevance of diagnosing structural chromosome abnormalities in couples with repeated miscarriage. Hum Reprod 2004;19(4):1013-7.

9. Franssen MT, Korevaar JC, Leschot NJ, Bossuyt PM, Knegt AC, Gerssen-Schoorl KB, et al. Selective chro- mosome analysis in couples with two or more miscarriages: case-control study. BMJ 2005;331(7509):13741.

10. Elghezal H, Hidar S, Mougou S, Khairi H, Saâd A. Prevalence of chromosomal abnormalities in couples with recurrent miscarriage. Fertil Steril 2007;88(3):721-3.

11. Pal S, Ma SO, Norhasimah M, Suhaida MA, Siti Mariam I, Ankathil R, Zilfalil BA. Chromosomal abnormalities and reproductive outcome in Malaysian couples with miscarriages. Singapore Med J 2009;50(10):1008-12.

12. Niroumanesh $S$, Mehdipour $P$, Farajpour A, Darvish S. A cytogenetic study of couples with repeated spontaneous abortions. Ann Saudi Med 2011;31(1):77-9.

13. Zhang Z, Gao H, Li S, Hong M, Liu R. Chromosomal abnormalities in patients with recurrent spontaneous abortions in northeast China. J Reprod Med 2011;56(7-8):321-4. 